TITLE:

\title{
Time-dependent density-functional tight-binding method with the third-order expansion of electron density.
}

\section{$\operatorname{AUTHOR}(S)$ :}

Nishimoto, Yoshio

\section{CITATION:}

Nishimoto, Yoshio. Time-dependent density-functional tight-binding method with the third-order expansion of electron density.. The Journal of chemical physics 2015, 143(9): 094108.

\section{ISSUE DATE:}

2015-09-04

URL:

http://hdl.handle.net/2433/202573

\section{RIGHT:}

(c) 2015 American Institute of Physics. This article may be downloaded for personal use only. Any other use requires prior permission of the author and the American Institute of Physics. The following article appeared in [J. Chem. Phys. 143, 094108 (2015)] and may be found at http://dx.doi.org/10.1063/1.4929926 


\section{AIP | $\begin{aligned} & \text { The Journal of } \\ & \text { Chemical Physics }\end{aligned}$}

\section{Time-dependent density-functional tight-binding method with the third-order expansion of electron density}

Yoshio Nishimoto

Citation: The Journal of Chemical Physics 143, 094108 (2015); doi: 10.1063/1.4929926

View online: http://dx.doi.org/10.1063/1.4929926

View Table of Contents: http://scitation.aip.org/content/aip/journal/jcp/143/9?ver=pdfcov

Published by the AIP Publishing

\section{Articles you may be interested in}

Lagrangian formulation with dissipation of Born-Oppenheimer molecular dynamics using the densityfunctional tight-binding method

J. Chem. Phys. 135, 044122 (2011); 10.1063/1.3605303

Torsional potentials and full-dimensional simulation of electronic absorption and fluorescence spectra of paraphenylene oligomers using the semiempirical self-consistent charge density-functional tight binding approach J. Chem. Phys. 129, 164905 (2008); 10.1063/1.2998523

Modeling carbon nanostructures with the self-consistent charge density-functional tight-binding method: Vibrational spectra and electronic structure of C 28, C 60 , and C 70

J. Chem. Phys. 125, 214706 (2006); 10.1063/1.2370877

Size-dependent properties of Zn m S n clusters: A density-functional tight-binding study

J. Chem. Phys. 123, 044311 (2005); 10.1063/1.1991853

Validation of the density-functional based tight-binding approximation method for the calculation of reaction energies and other data

J. Chem. Phys. 122, 114110 (2005); 10.1063/1.1871913

\section{AIP $\left.\right|_{\text {APL Photonics }}$}

APL Photonics is pleased to announce Benjamin Eggleton as its Editor-in-Chief

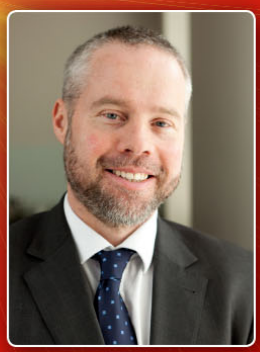




\title{
Time-dependent density-functional tight-binding method with the third-order expansion of electron density
}

\author{
Yoshio Nishimoto (西本佳央) ${ }^{\mathrm{a})}$ \\ Fukui Institute for Fundamental Chemistry, Kyoto University, 34-4 Takano Nishihiraki-cho, Sakyo-ku, \\ Kyoto 606-8103, Japan
}

(Received 1 July 2015; accepted 20 August 2015; published online 4 September 2015)

\begin{abstract}
We develop a formalism for the calculation of excitation energies and excited state gradients for the self-consistent-charge density-functional tight-binding method with the third-order contributions of a Taylor series of the density functional theory energy with respect to the fluctuation of electron density (time-dependent density-functional tight-binding (TD-DFTB3)). The formulation of the excitation energy is based on the existing time-dependent density functional theory and the older TD-DFTB2 formulae. The analytical gradient is computed by solving $\mathrm{Z}$-vector equations, and it requires one to calculate the third-order derivative of the total energy with respect to density matrix elements due to the inclusion of the third-order contributions. The comparison of adiabatic excitation energies for selected small and medium-size molecules using the TD-DFTB2 and TD-DFTB3 methods shows that the inclusion of the third-order contributions does not affect excitation energies significantly. A different set of parameters, which are optimized for DFTB3, slightly improves the prediction of adiabatic excitation energies statistically. The application of TD-DFTB for the prediction of absorption and fluorescence energies of cresyl violet demonstrates that TD-DFTB3 reproduced the experimental fluorescence energy quite well. (C) 2015 AIP Publishing LLC. [http://dx.doi.org/10.1063/1.4929926]
\end{abstract}

\section{INTRODUCTION}

Prediction of photochemical properties has been increasingly popular due to the advancement of time-dependent density functional theory (TD-DFT) ${ }^{1-6}$ Its reasonable computational cost and accuracy have given a large number of valuable theoretical insights. The low computational cost is partly attributed to algorithmic developments such as efficient matrix manipulations ${ }^{7,8}$ and analytical first ${ }^{9-11}$ and secondorder $^{12,13}$ geometrical derivative techniques.

Even with such advancement in TD-DFT, the computational demanding is still a heavy burden for researchers who wish to investigate large systems. An alternative method is the density-functional tight-binding (DFTB) method. ${ }^{14-17}$ The first generation of DFTB ${ }^{18}$ is based on the concept of extended Hückel method with parameters obtained from reference atomic DFT calculations. The total energy of DFTB is derived from that of DFT with the introduction of the tight-binding approach, and electron density is expanded with minimal Slater-type valence orbitals. This type of DFTB is called nonself-consistent-charge DFTB (NCC-DFTB or DFTB1) in which charge-charge interactions are neglected, and the energy for DFTB1 is obtained after single diagonalization of the secular equation. As the second generation of DFTB, Elstner et al. developed the self-consistent charge version of DFTB ${ }^{19}$ (SCC-DFTB or DFTB2) which includes the second-order terms of a Taylor series of the DFT total energy with respect to the fluctuation of electron density. In the framework of DFTB2, atomic charges are determined iteratively during SCC cycles, which are practically equivalent to self-consistent field

\footnotetext{
a)E-mail: nishimoto.yoshio@fukui.kyoto-u.ac.jp
}

(SCF) cycles, and properties such as energy, dipole moments, and gradients are to be easily calculated after self-consistency is fulfilled.

With DFTB2, the time-dependent version of DFTB2 (TD-DFTB2) was first introduced by Niehaus et al. ${ }^{20}$ in 2001. The formula for TD-DFTB is closely related to those for TDDFT; excitation energies are obtained by solving the Casida's equation. ${ }^{2,5}$ Later, the analytical gradient of TD-DFTB2 was developed by Heringer et $_{\text {al. }}{ }^{21}$ in 2007 based on the technique for TD-DFT ${ }^{9,10}$ utilizing the Z-vector method. ${ }^{22}$ These TDDFTB studies have shown that the accuracy of TD-DFTB is comparable with that of TD-DFT, while the computational cost is significantly reduced. Developments in TD-DFTB have enabled us to investigate excited state properties of silicon materials, ${ }^{23,24}$ the dynamics of microsolvated adenine, ${ }^{25}$ azobenzene, ${ }^{26}$ and cycloparaphenylenes. ${ }^{27}$

From a different scope of developments in DFTB, DFTB3 has recently been proposed. ${ }^{28-30}$ DFTB3 conceptually holds two independent improvements. While DFTB2 includes a Taylor series of the DFT energy up to second order contributions, DFTB3 includes the third-order contributions, and this is the first improvement in DFTB3. They are responsible for changing chemical hardness, which is related to Hubbard parameters, of an atom depending on the charge on it. The necessity of the flexible chemical hardness comes from the fact that chemical hardness depends on the charge on an atom. ${ }^{28,31}$ Another improvement in DFTB3 is the use of a damping function for the $\gamma$ function for pairs which include a hydrogen atom. The $\gamma$ function used in DFTB2 and DFTB3 ideally approaches the Hubbard value at a short distance, but it is not the case for hydrogen atoms due to an inconsistency between the atomic size and the chemical 
hardness. ${ }^{28,31}$ Previous investigations ${ }^{17,30,32-34}$ have proved that DFTB 3 is systematically superior to DFTB2 in describing hydrogen-bonded and polar systems.

In spite of a number of developments in parameters for DFTB3 at the ground state (GS), ${ }^{30,32,35-37}$ the formulation and the performance of the time-dependent version of DFTB3 (TD-DFTB3) are still missing. In this study, we derive the excitation energy with the restricted TD-DFTB3, following the previous TD-DFTB2 formula, and its analytical firstorder geometrical derivative. The performance of TD-DFTB2 and TD-DFTB3 is also presented by comparing adiabatic excitation energies and absorption and fluorescence energies of a small dye in explicit water molecules.

\section{THEORY}

\section{A. DFTB3 at the ground state}

The total energy of DFTB3, $E$, is given by

$$
\begin{aligned}
E= & \sum_{\sigma} \sum_{i} n_{i \sigma} \sum_{\mu \nu} C_{\mu i \sigma} C_{v i \sigma} H_{\mu \nu}^{0}+\sum_{A>B} E_{A B}^{\mathrm{rep}} \\
& +\frac{1}{2} \sum_{A, B} \gamma_{A B} \Delta q_{A} \Delta q_{B} \\
& +\frac{1}{6} \sum_{A, B}\left(\Gamma_{A B} \Delta q_{A}+\Gamma_{B A} \Delta q_{B}\right) \Delta q_{A} \Delta q_{B}+E^{\mathrm{spin}},
\end{aligned}
$$

where $\mu$ and $\nu$ (for latter use, $\kappa, \lambda, \phi$, and $\chi$ ) are the indices for atomic orbitals (AOs), $A$ and $B(C, D$, and $E$ ) are those for atoms. The occupation number of $i$ th molecular orbital (MO) is represented as $n_{i \sigma}$, and it is either 0 or 1 for virtual and occupied orbitals in the present work. We only treat the case for restricted electronic configuration in this study, but spin indices $\sigma, \tau$, and $v$, which are either $\alpha$-or $\beta$-orbital/electron are explicitly denoted. The fifth term in Eq. (1) represents the spin interaction energy defined at DFTB2 level ${ }^{38}$ explicitly, but this term is always zero in this study due to restricted electronic configuration. As usual, the repulsive energy, $E_{A B}^{\mathrm{rep}}$, and the non-perturbed zeroth-order Hamiltonian, $H_{\mu \nu}^{0}$, are precomputed with reference DFT calculations. The Coulombic interaction is described with the third and fourth terms in Eq. (1) where $\gamma_{A B}$ and $\Gamma_{A B}$ depend on the distance between two atoms $A$ and $B$ and their Hubbard values. As for $\Gamma_{A B}$, it also depends on the derivative of the Hubbard value with respect to atomic Mulliken charges $\Delta q_{A}$ calculated as $\Delta q_{A}=q_{A}-q_{A}^{0}$, where $q_{A}$ is the Mulliken population on atom $A$, defined as

$$
q_{A}=\sum_{\sigma} \sum_{\mu \in A} \sum_{\nu} D_{\mu \nu \sigma} S_{\mu \nu}
$$

where $D_{\mu \nu \sigma}$ is the usual density matrix, and $q_{A}^{0}$ is the number of electrons on atom $A$ in neutral. One of two developments in DFTB3 is the modification of $\gamma$ and $\Gamma$ values to $\gamma^{\mathrm{h}}$ and $\Gamma^{\mathrm{h}}$ for pairs that contain a hydrogen atom. However, we will not explicitly make a distinction between the standard $\gamma$ and $\Gamma$ and $\gamma^{\mathrm{h}}$ and $\Gamma^{\mathrm{h}}$ values. In practical calculations, one can simply replace $\gamma$ and $\Gamma$ with $\gamma^{\mathrm{h}}$ and $\Gamma^{\mathrm{h}}$, and there is no need to modify the code other than the calculation of $\gamma$ and $\Gamma$. Despite $\gamma$ and $\Gamma$ are used for the derivation hereafter, all equations are applicable for the case with and without the damping function.
The derivative of the total energy (Eq. (1)) with respect to a density matrix element leads to the Hamiltonian (Fock) matrix element,

$$
H_{\mu \nu}=H_{\mu \nu}^{0}+S_{\mu \nu} \Omega_{A B},
$$

where $\mu \in A$ and $v \in B$, and $S_{\mu \nu}$ is the overlap matrix element in AO basis. The shift contribution $\Omega_{A B}$ describes the Coulombic interaction,

$$
\begin{aligned}
\Omega_{A B}= & \frac{1}{2} \sum_{C}\left(\gamma_{A C}+\gamma_{B C}\right) \Delta q_{C} \\
& +\frac{1}{6} S_{\mu \nu} \sum_{C}\left\{2\left(\Gamma_{A C} \Delta q_{A}+\Gamma_{B C} \Delta q_{B}\right)\right. \\
& \left.+\left(\Gamma_{C A}+\Gamma_{C B}\right) \Delta q_{C}\right\} \Delta q_{C} .
\end{aligned}
$$

Since the spin contributions in $\Omega_{A B}$ are zero with restricted wavefunction, we omit it. Overlap matrix elements are also precomputed as non-perturbed Hamiltonian elements are done so. Obviously, the Hamiltonian matrix depends on the current density of the system under consideration, so the MO coefficients $\left(C_{\mu i \sigma}\right)$ in density matrix have to be determined self-consistently.

For a seamless connection with other $a b$ initio methods (e.g., Hartree-Fock or DFT), Eq. (1) can be written in a so-called density matrix formalism,

$$
E=\frac{1}{2} \sum_{\sigma} \sum_{\mu \nu} D_{\mu \nu \sigma}\left(\tilde{H}_{\mu \nu}^{\text {core }}+\tilde{F}_{\mu \nu}\right)+E^{3 \mathrm{rd}}+\tilde{E}^{\text {rep }},
$$

where

$$
\tilde{H}_{\mu \nu}^{\mathrm{core}}=H_{\mu \nu}^{0}-\frac{1}{2} S_{\mu \nu} \sum_{C}\left(\gamma_{A C}+\gamma_{B C}\right) q_{C}^{0}
$$

and

$$
\tilde{F}_{\mu \nu}=\tilde{H}_{\mu \nu}^{\mathrm{core}}+\frac{1}{2} S_{\mu \nu} \sum_{C}\left(\gamma_{A C}+\gamma_{B C}\right) q_{C} .
$$

$E^{3 \mathrm{rd}}$ corresponds to the fourth term in Eq. (1), and $\tilde{E}^{\text {rep }}$ is defined as

$$
\tilde{E}^{\mathrm{rep}}=\sum_{A>B}\left(E_{A B}^{\mathrm{rep}}+\gamma_{A B} q_{A}^{0} q_{B}^{0}\right) .
$$

Because $E^{3 \text { rd }}$ depends on the cubic of density fluctuation and comes from the third-order derivative of exchange-correlation contributions, ${ }^{30}$ we cannot straightforwardly incorporate it with $\tilde{H}_{\mu \nu}$ and $\tilde{F}_{\mu \nu}$ as a previous study with DFTB2 did. ${ }^{39}$ The third-order contributions may be seen as a pseudo-exchangecorrelation term.

The gradient of the DFTB3 energy with respect to the geometrical displacement of the coordinate $x$ of atom $\alpha, R_{\alpha x}$ ( $x$ in the derivatives can be replaced with $y$ or $z$ ), is

$$
\begin{aligned}
\frac{\partial E}{\partial R_{\alpha x}}= & \sum_{\sigma} \sum_{i} n_{i \sigma} \sum_{A \neq \alpha} \sum_{\mu \in A} \sum_{v \in \alpha} C_{\mu i \sigma} C_{\nu i \sigma} \\
& \times\left[2 \frac{\partial H_{\mu \nu}^{0}}{\partial R_{\alpha x}}-2 \varepsilon_{i \sigma} \frac{\partial S_{\mu \nu}}{\partial R_{\alpha x}}+2 \frac{\partial S_{\mu \nu}}{\partial R_{\alpha x}} \Omega_{A \alpha}\right] \\
& +\sum_{A \neq \alpha} \frac{\partial E_{A \alpha}^{\text {rep }}}{\partial R_{\alpha x}}+\Delta q_{\alpha} \sum_{A \neq \alpha} \Delta q_{A} \frac{\partial \gamma_{A \alpha}}{\partial R_{\alpha x}} \\
& +\frac{1}{3} \Delta q_{\alpha} \sum_{A \neq \alpha} \Delta q_{A}\left(\Delta q_{A} \frac{\partial \Gamma_{A \alpha}}{\partial R_{\alpha x}}+\Delta q_{\alpha} \frac{\partial \Gamma_{\alpha A}}{\partial R_{\alpha x}}\right) .
\end{aligned}
$$


In a density matrix formalism, one can express Eq. (9) as

$$
\begin{aligned}
\frac{\partial E}{\partial R_{\alpha x}}= & \sum_{\sigma} \sum_{\mu \nu} D_{\mu \nu \sigma}\left(\frac{\partial \tilde{H}_{\mu \nu}^{\text {core }}}{\partial R_{\alpha x}}+\frac{\partial \tilde{F}_{\mu \nu}}{\partial R_{\alpha x}}\right) \\
& -\sum_{\sigma} \sum_{\mu \nu} W_{\mu \nu \sigma} \frac{\partial S_{\mu \nu}}{\partial R_{\alpha x}}+\frac{\partial E^{3 \mathrm{rd}}}{\partial R_{\alpha x}}+\frac{\partial \tilde{E}^{\mathrm{rep}}}{\partial R_{\alpha x}}
\end{aligned}
$$

where

$$
\begin{gathered}
\frac{\partial \tilde{H}_{\mu \nu}^{\text {core }}}{\partial R_{\alpha x}}=\frac{\partial H_{\mu \nu}^{0}}{\partial R_{\alpha x}}-\frac{1}{2} \frac{\partial S_{\mu \nu}}{\partial R_{\alpha x}} \sum_{C}\left(\gamma_{A C}+\gamma_{B C}\right) q_{C}^{0} \\
-\frac{1}{2} S_{\mu \nu} \sum_{C}\left(\frac{\partial \gamma_{A C}}{\partial R_{\alpha x}}+\frac{\partial \gamma_{B C}}{\partial R_{\alpha x}}\right) q_{C}^{0}
\end{gathered}
$$

and

$$
\begin{aligned}
\frac{\partial \tilde{F}_{\mu \nu}}{\partial R_{\alpha x}}= & \frac{\partial \tilde{H}_{\mu \nu}^{\mathrm{core}}}{\partial R_{\alpha x}}+\frac{\partial S_{\mu \nu}}{\partial R_{\alpha x}} \sum_{C}\left(\gamma_{A C}+\gamma_{B C}\right) q_{C} \\
& +\frac{1}{2} S_{\mu \nu} \sum_{C}\left(\frac{\partial \gamma_{A C}}{\partial R_{\alpha x}}+\frac{\partial \gamma_{B C}}{\partial R_{\alpha x}}\right) q_{C} .
\end{aligned}
$$

$W_{\mu \nu \sigma}$ is the usual energy-weighted density matrix, and $\left(\partial E^{3 \mathrm{rd}}\right) /\left(\partial R_{\alpha x}\right)$ represents

$$
\begin{aligned}
\frac{\partial E^{3 \mathrm{rd}}}{\partial R_{\alpha x}}= & \frac{1}{6} \sum_{\sigma} \sum_{\mu \nu} D_{\mu \nu \sigma} \frac{\partial S_{\mu \nu}}{\partial R_{\alpha x}} \sum_{C}\left\{2\left(\Gamma_{A C} \Delta q_{A}+\Gamma_{B C} \Delta q_{B}\right)\right. \\
& \left.+\left(\Gamma_{C A}+\Gamma_{C B}\right) \Delta q_{C}\right\} \Delta q_{C} \\
& +\frac{1}{6} \sum_{A, B} \Delta q_{A} \Delta q_{B}\left(\Delta q_{A} \frac{\partial \Gamma_{A B}}{\partial R_{\alpha x}}+\Delta q_{B} \frac{\partial \Gamma_{B A}}{\partial R_{\alpha x}}\right) .
\end{aligned}
$$

\section{B. Excited state with the DFTB3 method}

Excitation energy $\omega$ and excitation vectors $\mathbf{X}$ and $\mathbf{Y}$ can be obtained by solving the non-Hermitian eigenvalue problem,

$$
\left(\begin{array}{ll}
\mathbf{A} & \mathbf{B} \\
\mathbf{B} & \mathbf{A}
\end{array}\right)\left(\begin{array}{l}
\mathbf{X} \\
\mathbf{Y}
\end{array}\right)=\omega\left(\begin{array}{cc}
\mathbf{1} & \mathbf{0} \\
\mathbf{0} & \mathbf{- 1}
\end{array}\right)\left(\begin{array}{l}
\mathbf{X} \\
\mathbf{Y}
\end{array}\right),
$$

where matrix elements of $\mathbf{A}$ and $\mathbf{B}$ are defined as

$$
A_{i a \sigma, j b \tau}=\delta_{\sigma \tau} \delta_{a b} \delta_{i j}\left(\varepsilon_{a \sigma}-\varepsilon_{i \sigma}\right)+K_{i a \sigma, j b \tau}
$$

and

$$
B_{i a \sigma, j b \tau}=K_{i a \sigma, b j \tau},
$$

where $\varepsilon_{i \sigma}$ is the eigenvalue of $i$ th $\mathrm{MO}$, and the element of the coupling matrix, $K_{i a \sigma, j b \tau}$, is given as

$$
K_{i a \sigma, j b \tau}=\frac{\partial^{2} E}{\partial D_{i a \sigma} \partial D_{j b \tau}} .
$$

In this work, $i, j, \ldots$ and $a, b, \ldots$ label occupied and virtual orbitals, respectively, and for latter use, $p, q, \ldots$ label general MOs. The coupling matrix is the second-order derivative of the total energy with respect to density matrix elements. In order to compute the coupling matrix element, it is convenient to introduce the four-index kernels which represent the charge and spin interaction terms for DFTB, ${ }^{40}$

$$
K_{\mu \nu \sigma, \kappa \lambda \tau}=\frac{\partial^{2} E}{\partial D_{\mu \nu \sigma} \partial D_{\kappa \lambda \tau}}=\hat{j}_{\mu \nu \sigma, \kappa \lambda \tau}+\hat{k}_{\mu \nu \sigma, \kappa \lambda \tau} .
$$

We note that these matrix elements for TD-DFTB $2^{40}$ are

$$
\hat{j}_{\mu \nu \sigma, \kappa \lambda \tau}^{\mathrm{DFTB} 2}=\frac{1}{4} S_{\mu \nu} S_{\kappa \lambda}\left(\gamma_{A C}+\gamma_{B C}+\gamma_{A D}+\gamma_{B D}\right)
$$

and

$$
\begin{aligned}
\hat{k}_{\mu \nu \sigma, \kappa \lambda \tau}^{\mathrm{DFTB} 2}= & \frac{1}{4} S_{\mu \nu} S_{\kappa \lambda}\left(M_{A} \delta_{A C}+M_{B} \delta_{B C}\right. \\
& \left.+M_{A} \delta_{A D}+M_{B} \delta_{B D}\right),
\end{aligned}
$$

where $\mu \in A, v \in B, \kappa \in C$, and $\lambda \in D$, and $M_{A}$ represents the spin constant of atom $A$ which is also obtained from reference DFT calculations. For TD-DFTB3, $\hat{j}_{\mu \nu \sigma, \kappa \lambda \tau}$ has to be reformulated as

$$
\begin{aligned}
\hat{j}_{\mu \nu \sigma, \kappa \lambda \tau}^{\mathrm{DFTB} 3}= & \hat{j}_{\mu \nu \sigma, \kappa \lambda \tau}^{\mathrm{DFTB} 2}+\frac{1}{6} S_{\mu \nu} S_{\kappa \lambda} \\
& \times\left[\left\{\left(\Gamma_{A C}+\Gamma_{A D}\right) \Delta q_{A}+\left(\Gamma_{B C}+\Gamma_{B D}\right) \Delta q_{B}\right\}\right. \\
& +\left\{\left(\Gamma_{C A}+\Gamma_{C B}\right) \Delta q_{C}+\left(\Gamma_{D A}+\Gamma_{D B}\right) \Delta q_{D}\right\} \\
& +\sum_{E}\left\{\Gamma_{A E}\left(\delta_{A C}+\delta_{A D}\right)\right. \\
& \left.\left.+\Gamma_{B E}\left(\delta_{B C}+\delta_{B D}\right)\right\} \Delta q_{E}\right] .
\end{aligned}
$$

In order to derive the spin interaction term needed for singlet-triplet excitation in the framework of DFTB3, we need to compute the second-order derivative of the DFTB energy with respect to spin density. The spin-polarized contributions in DFTB $^{38}$ are defined as

$$
E^{\mathrm{spin}}=\frac{1}{2} \sum_{A} \sum_{\ell \in A} \sum_{\ell^{\prime} \in A} p_{A \ell} p_{A \ell^{\prime}} W_{A \ell \ell^{\prime}},
$$

where $p_{A \ell}$ is the shell( $(\ell)$-resolved spin density, and $W_{A \ell \ell^{\prime}}$ is a spin constant calculated using Janak's theorem. ${ }^{41}$ It is clear from the derivation by Köhler et al. that Eq. (22) is derived within the framework of DFTB2. The extension of the spin-polarized contribution to the DFTB3 framework is considered to require an additional parameter, the derivative of spin constants with respect to spin density, but the development in this direction is far beyond the scope of this study. For this reason, we will not consider DFTB3 contributions for singlettriplet excitation explicitly, but only DFTB2 contribution (Eq. (20)) in our derivation.

In order to obtain excitation energies and vectors, we need to diagonalize the matrix operator of the left-hand in Eq. (14). Since the size of the matrix operator becomes huge for large systems, it is usually diagonalized iteratively. ${ }^{42}$ Practically, we construct the four-index matrices $(\mathbf{A}+\mathbf{B})$ and $(\mathbf{A}-\mathbf{B})$ defined as

$(A+B)_{i a \sigma, j b \tau}=\delta_{\sigma \tau} \delta_{a b} \delta_{i j}\left(\varepsilon_{a \sigma}-\varepsilon_{i \sigma}\right)+2 K_{i a \sigma, j b \tau}$

and

$$
(A-B)_{i a \sigma, j b \tau}=\delta_{\sigma \tau} \delta_{a b} \delta_{i j}\left(\varepsilon_{a \sigma}-\varepsilon_{i \sigma}\right),
$$

which are known as orbital rotation Hessians. There has been discussion of the character of the $(\mathbf{A}-\mathbf{B})$ matrix for TDDFTB..$^{40}$ In TD-DFT, the matrix becomes diagonal with a pure (i.e., without Hartree-Fock exchange) exchange-correlation functional such as generalized gradient approximation functionals including the Perdew, Burke, and Ernzerhof (PBE) which is used for generating the electronic part of DFTB parameters. As DFTB is derived from a DFT formalism 
without Hartree-Fock exchange, we assume that $(\mathbf{A}-\mathbf{B})$ matrix for TD-DFTB3 is strictly diagonal.

By neglecting the matrix $\mathbf{B}$, we only have to evaluate Eq. (15). This approximation is known as the Tamm-Dancoff approximation (TDA), ${ }^{43}$ and since the difference is only the scaling of $K_{i a \sigma, j b \tau}$, we can introduce TDA for TD-DFTB with a slight modification. Although some advantages ${ }^{43}$ have been recognized in TDA, we do not use TDA in this study. Since the real value of TDA for TD-DFT calculations is the case with hybrid or range-separated functionals with which $(\mathbf{A}-\mathbf{B})$ matrix is not diagonal, we think that TDA does not change the overall conclusions in this study.

\section{Analytical excitation energy gradient of TD-DFTB3}

Concise derivations for TD-DFT and TD-DFTB2 can be found in the previous literature. ${ }^{9,21,44}$ Solving the nonHermitian eigenvalue problem given as Eq. (14) corresponds to finding the stationary points of the functional defined as

$$
\begin{aligned}
G[\mathbf{X}, \mathbf{Y}, \omega]= & \frac{1}{2} \sum_{i a \sigma j b \tau}\left\{(X+Y)_{i a \sigma}(A+B)_{i a \sigma, j b \tau}(X+Y)_{j b \tau}\right. \\
& \left.+(X-Y)_{i a \sigma}(A-B)_{i a \sigma, j b \tau}(X-Y)_{j b \tau}\right\} \\
& -\omega\left\{\sum_{i a \sigma}(X+Y)_{i a \sigma}(X-Y)_{i a \sigma}-1\right\}
\end{aligned}
$$

where the second term enforces the orthonormality, ${ }^{3}$ and the excitation energy $\omega$ is given as

$$
\begin{aligned}
\omega= & \frac{1}{2} \sum_{i a \sigma j b \tau}\left\{(X+Y)_{i a \sigma}(A+B)_{i a \sigma, j b \tau}(X+Y)_{j b \tau}\right. \\
& \left.+(X-Y)_{i a \sigma}(A-B)_{i a \sigma, j b \tau}(X-Y)_{j b \tau}\right\} .
\end{aligned}
$$

Since excitation vectors are variationally optimized, we do not need to calculate derivatives of $(\mathbf{X}+\mathbf{Y})$ and $(\mathbf{X}-\mathbf{Y})$ for excitation energy gradient, but we need to involve derivatives of MO coefficients, contrary to gradient calculations at the ground state. Although one can obtain them explicitly by solving the coupled-perturbed DFTB equations, ${ }^{45}$ it is computationally demanding, because it requires solving a set of $3 N$ linear equations, where $N$ is the number of atoms. Such an explicit evaluation can be avoided by utilizing the Z-vector method. ${ }^{9,22}$ Solving Z-vector equations corresponds to finding the Lagrange multipliers of the auxiliary functional,

$$
\begin{aligned}
L[\mathbf{X}, \mathbf{Y}, \omega, \mathbf{C}, \mathbf{Z}, \mathbf{W}] & \frac{1}{2} \sum_{i a \sigma j b \tau}\left\{(X+Y)_{i a \sigma}(A+B)_{i a \sigma, j b \tau}(X+Y)_{j b \tau}\right. \\
& \left.+(X-Y)_{i a \sigma}(A-B)_{i a \sigma, j b \tau}(X-Y)_{j b \tau}\right\} \\
& -\omega\left\{\sum_{i a \sigma}(X+Y)_{i a \sigma}(X-Y)_{i a \sigma}-1\right\} \\
& +\sum_{i a \sigma} Z_{i a \sigma} H_{i a \sigma}-\sum_{p q \sigma, p \leq q} W_{p q \sigma}\left(S_{p q \sigma}-\delta_{p q}\right),
\end{aligned}
$$

and Eq. (27) has to be stationary with respect to changes in MO coefficients,

$$
\frac{\partial L}{\partial C_{\mu p \sigma}}=0
$$

Some supplementary equations are given in Appendix A, and we simply introduce new terms in TD-DFTB3. In order to evaluate the vector $\mathbf{Z}$, we need to solve the following equation:

$$
\sum_{j b \tau}(A+B)_{i a \sigma, j b \tau} Z_{j b \tau}=-R_{i a \sigma},
$$

where $R_{i a \sigma}$ is obtained as the difference of Eqs. (A5) and (A6),

$$
\begin{aligned}
R_{i a \sigma}= & \sum_{b}\left\{(X+Y)_{i b \sigma} H_{a b \sigma}^{+}[(\mathbf{X}+\mathbf{Y})]\right\}+H_{i a \sigma}^{+}[\mathbf{T}] \\
& +2 \sum_{j b \tau k c v} \hat{g}_{i a \sigma, j b \tau, k c v}(X+Y)_{j b \tau}(X+Y)_{k c v} \\
& -\sum_{j}\left\{(X+Y)_{j a \sigma} H_{j i \sigma}^{+}[(\mathbf{X}+\mathbf{Y})]\right\},
\end{aligned}
$$

where the vector $\mathbf{T}$ is given in Appendix A, and for an arbitrary vector $\mathbf{V}$,

$$
H_{p q \sigma}^{+}[\mathbf{V}]=\sum_{r s \tau} K_{p q \sigma, r s \tau} V_{r s \tau} .
$$

Note that $H_{p q \sigma}^{-}[\mathbf{V}]$ found in TD-DFT derivations is always zero for both TD-DFTB2 and TD-DFTB3 unless an alternative formulation ${ }^{40}$ is used. The third term in Eq. (30) represents the third-order derivative of the DFTB energy, and it did not appear in the derivation for the TD-DFTB2 analytical gradient. ${ }^{21}$ This complication can be understood by comparing the $\hat{j}_{\mu \nu \sigma, \kappa \lambda \tau}$ for TD-DFTB2 (Eq. (19)) and TD-DFTB3 (Eq. (21)). The former becomes trivial after differentiating with an additional density matrix element, but the latter does not, because Eq. (21) still contains Mulliken charges, which depends on the density matrix.

The third-order derivative in Eq. (30) can be explicitly written by differentiating the total energy with three density matrix elements. In $\mathrm{AO}$ representation, the derivative is

$$
\begin{aligned}
\hat{g}_{\mu \nu \sigma, \kappa \lambda \tau, \phi \chi v}= & \frac{1}{12} S_{\mu \nu} S_{\kappa \lambda} S_{\phi \chi} \\
& \times \sum_{E}\left\{\Gamma_{A E}\left(\delta_{A C}+\delta_{A D}\right)+\Gamma_{B E}\left(\delta_{B C}+\delta_{B D}\right)\right\} \\
& \times\left(\delta_{\phi \in E}+\delta_{\chi \in E}\right) .
\end{aligned}
$$

However, in practice, it is convenient to define a two-third transformed matrix,

$$
\begin{aligned}
\sum_{\kappa \lambda \tau \phi \chi v} \hat{g}_{\mu v \sigma, \kappa \lambda \tau, \phi \chi v}(X+Y)_{\kappa \lambda \tau}(X+Y)_{\phi \chi v} \\
=\frac{1}{3} S_{\mu v} \sum_{C}\left\{2\left(\Gamma_{A C} q_{A}^{X Y}+\Gamma_{B C} q_{B}^{X Y}\right) q_{C}^{X Y}\right. \\
\left.\quad+\left(\Gamma_{C A}+\Gamma_{C B}\right)\left(q_{C}^{X Y}\right)^{2}\right\}
\end{aligned}
$$

where

$$
q_{A}^{X Y}=\sum_{\sigma} \sum_{\mu \in A} \sum_{\nu}(X+Y)_{\mu \nu \sigma} S_{\mu \nu} .
$$

Once the Z-vector equation (Eq. (29)) is solved, we obtain two Lagrange multipliers, $\mathbf{Z}$ and $\mathbf{W}$. By defining the relaxed one-particle difference density matrix, $\mathbf{P}=\mathbf{T}+\mathbf{Z}$, and the effective two-particle difference density matrix $\hat{\Gamma}_{\mu \nu \sigma, \kappa \lambda \tau}$,

$$
\hat{\Gamma}_{\mu \nu \sigma, \kappa \lambda \tau}=(X+Y)_{\mu \nu \sigma}(X+Y)_{\kappa \lambda \tau}+P_{\mu \nu \sigma} D_{\kappa \lambda \tau},
$$


the excitation energy gradient is finally given as

$$
\begin{aligned}
\frac{\partial \omega}{\partial R_{\alpha x}}= & \sum_{\mu \nu \sigma} \frac{\partial H_{\mu \nu}^{0}}{\partial R_{\alpha x}} P_{\mu \nu \sigma}-\sum_{\mu \nu \sigma} \frac{\partial S_{\mu \nu}}{\partial R_{\alpha x}} W_{\mu \nu \sigma} \\
& +\frac{1}{2} \sum_{\mu \nu \sigma \kappa \lambda \tau} \frac{\partial K_{\mu \nu \sigma, \kappa \lambda \tau}}{\partial R_{\alpha x}} \hat{\Gamma}_{\mu \nu \sigma, \kappa \lambda \tau} .
\end{aligned}
$$

Eq. (36) for singlet-singlet and singlet-triplet excitations is explicitly given in Appendix B. The TD-DFTB3, as well as the TD-DFTB2, with and without TDA was implemented in GAMESS-US ${ }^{46}$ whose TD-DFT module had been developed by Chiba et al. ${ }^{10}$ Comparison with analytical and numerical gradients for several excited states with glycine molecule shows that our analytical gradient reproduces numerical gradients in six decimal place accuracy.

\section{COMPUTATIONAL DETAILS}

We used the $\mathrm{MIO}^{19,30,47}$ and $3 \mathrm{OB}^{32,35}$ sets of DFTB parameters $^{48}$ in TD-DFTB2 and TD-DFTB3 calculations. Spin constants $M_{A}=W_{\text {Ass }}$ used in singlet-triplet excitation are also taken from the same place. In order to access the performance of TD-DFTB2 and TD-DFTB3, we consider two and three sets of TD-DFTB2 and TD-DFTB3 calculations. They are referred as TD-DFTB2/MIO, TDDFTB2/3OB, TD-DFTB3/MIO/calc, TD-DFTB3/MIO/fit, and TD-DFTB3/3OB; the capital letters after the first slash specify the employed parameter set, and the letters after the second slash, if any, do the employed Hubbard derivatives. The difference of methods is given in Ref. 32, and the parameter $\zeta$ used for the damping function and Hubbard derivatives for DFTB3 are also provided in the same reference and Refs. 30 and 35. The Hubbard derivative for $\mathrm{S}$ atom for TD-DFTB3/MIO/fit was missing, so we simply used the one for TD-DFTB3/3OB. All DFTB calculations were performed without symmetry constraints.

The performance of TD-DFTB2 and TD-DFTB3 is discussed based on adiabatic excitation energies for selected molecules listed in Refs. 9, 49, and 50. Molecules for which we do not have parameters are excluded. The estimation of the zero point vibration energy requires to compute the second-order derivatives at the ground and the excited states. We analytically calculated them for the ground state, while numerically for excited states with a step size of $1.0 \times 10^{-4}$ a.u. with analytical gradients.

We briefly compare wall-clock timings of TDDFTB2/MIO and TD-DFTB3/3OB. For the demonstration, we chose a trans-polyacetylene $\left(\mathrm{C}_{400} \mathrm{H}_{402}\right)$ which contained 2002 basis functions. The five lowest excitation energies were calculated, and the first excitation vectors were used in the gradient calculation. The computation of the energy gradient at an excited state mainly consists of three steps: SCF calculation at the GS, solving the Casida's equation to find excitation energies (ES: excited state), and solving Z-vector equations followed by the computation of gradient (ES-GRAD). Table I shows the respective timings and the numbers of iterations until convergence. Clearly, additional computational cost for TD-DFTB3 compared with TD-DFTB2 is almost negligible.
TABLE I. Wall-clock timings (unit in s) and numbers of iterations to obtain the five lowest excitation energies and the excited state gradient at the first excited state for $\mathrm{C}_{400} \mathrm{H}_{402}$ with TD-DFTB2/MIO and TD-DFTB3/3OB. Calculations were performed with one CPU core of Xeon E5-1620 v3 (3.50 GHz).

\begin{tabular}{lcc}
\hline \hline Step & Wall-clock timings & $\begin{array}{c}\text { Number of } \\
\text { iterations }\end{array}$ \\
\hline & TD-DFTB2 & \\
\hline GS & 213.80 & 17 \\
ES & 875.97 & 19 \\
ES-GRAD & 42.63 & 3 \\
& TD-DFTB3 & 17 \\
\hline GS & 213.82 & 18 \\
ES & 819.92 & 3 \\
ES-GRAD & 45.54 & \\
\hline \hline
\end{tabular}

The timings per iteration during ES calculations for TDDFTB2 and TD-DFTB3 are 46.10 and $45.55 \mathrm{~s}$, respectively. However, considering that the number of trial vectors required for convergence is different in two calculations, it is more meaningful to compare the timings per trial vector. The numbers of trial vectors used in the TD-DFTB2 and TDDFTB3 calculations are 89 and 83 , respectively, so the timings per trial vector are 9.84 and $9.88 \mathrm{~s}$. The computational efficiency of TD-DFTB3 is actually very similar to that of TD-DFTB2.

\section{RESULTS AND DISCUSSION}

\section{A. Performance of TD-DFTB2 and TD-DFTB3}

Calculated adiabatic excitation energies are summarized in Table II with root-mean-square (RMS) and maximum deviations. Our TD-DFTB2/MIO results are slightly different from those by Heringer et al. ${ }^{21}$ and we think that parameters and spin constants used in our calculations are slightly different from those used in Ref. 21.

Table II shows that adiabatic excitation energies calculated with TD-DFTB2/MIO, TD-DFTB3/MIO/calc, and TD-DFTB3/MIO/fit are overall very similar, and the difference is not more than $0.04 \mathrm{eV}$. The difference between RMS and maximum deviations calculated with all items between these methods is also very small. Additionally, the difference in energies with TD-DFTB2/3OB and TD-DFTB3/3OB is small as well. This indicates that the extension to TD-DFTB3 from TD-DFTB2 affects the excited state properties very little. It is because the DFTB3 model does not affect the levels of eigenvalues (orbital energies) significantly. The DFTB method is originally derived from a Taylor expansion of the total energy of DFT. The nonself-charge-consistent version of DFTB, DFTB1, already includes the zeroth Hamiltonian contributions which are calculated from atomic DFT calculations. Although DFTB2 and DFTB3 take the charge fluctuation into consideration with a better model, it is a perturbative effect, and orbital energies are already determined mostly with the DFTB1 level. Moreover, some excitations are described with a single orbital transition character whose excitation energy is simply determined by the 
TABLE II. Adiabatic excitation energies (unit in eV) from experiment and those calculated with five TD-DFTB methods. RMS and maximum (MAX) deviations (unit in $\mathrm{eV}$ ) for singlet-singlet $(\mathrm{S})$ and singlet-triplet $(\mathrm{T})$ excitations and all items are shown at the bottom. Values in parentheses do not include zero point vibration corrections.

\begin{tabular}{|c|c|c|c|c|c|c|c|}
\hline System & State & Experiment & TD-DFTB2/MIO & TD-DFTB3/MIO/calc & TD-DFTB3/MIO/fit & TD-DFTB2/3OB & TD-DFTB3/3OB \\
\hline \multirow[t]{2}{*}{$\mathrm{N}_{2}$} & ${ }^{3} \Pi_{\mathrm{g}}$ & (7.39) & (7.89) & (7.89) & (7.89) & $(8.02)$ & $(8.02)$ \\
\hline & ${ }^{1} \Pi_{\mathrm{g}}^{\circ}$ & (8.59) & (7.89) & (7.89) & (7.89) & $(8.02)$ & $(8.02)$ \\
\hline \multirow[t]{2}{*}{$\mathrm{CO}$} & ${ }^{3} \Pi$ & (6.04) & (7.63) & (7.63) & (7.63) & (7.32) & (7.32) \\
\hline & ${ }^{1} \Pi$ & $(8.07)$ & $(7.63)$ & $(7.63)$ & $(7.63)$ & $(7.32)$ & $(7.32)$ \\
\hline $\mathrm{SO}_{2}$ & ${ }^{3} B_{1}$ & 3.19 & 3.09 & 3.09 & 3.08 & 2.80 & 2.79 \\
\hline $\mathrm{CS}_{2}$ & ${ }^{3} A_{2}$ & 3.25 & 2.79 & 2.78 & 2.78 & 3.24 & 3.23 \\
\hline $\mathrm{HCN}$ & ${ }^{1} A^{\prime \prime}$ & 6.48 & 6.37 & 6.38 & 6.38 & 6.16 & 6.28 \\
\hline $\mathrm{HCP}$ & ${ }^{1} A^{\prime \prime}$ & 4.31 & 3.66 & 3.66 & 3.66 & 3.62 & 3.63 \\
\hline $\mathrm{C}_{2} \mathrm{H}_{2}$ & ${ }^{1} A_{\mathrm{u}}$ & 5.23 & 4.71 & 4.74 & 4.74 & 4.69 & 4.72 \\
\hline \multirow[t]{2}{*}{$\mathrm{CH}_{2} \mathrm{O}$} & ${ }^{1} A^{\prime \prime}$ & 3.49 & 3.76 & 3.77 & 3.76 & 3.65 & 3.66 \\
\hline & ${ }^{3} A^{\prime \prime}$ & 3.12 & 3.66 & 3.67 & 3.66 & 3.55 & 3.56 \\
\hline \multirow[t]{2}{*}{$\mathrm{CH}_{2} \mathrm{~S}$} & ${ }^{1} A_{2}$ & 2.03 & 1.74 & 1.73 & 1.74 & $2.00^{\mathrm{a}}$ & $1.98^{\mathrm{a}}$ \\
\hline & ${ }^{3} A^{\prime \prime}$ & 1.80 & 1.74 & 1.73 & 1.73 & 2.01 & 2.00 \\
\hline trans $-(\mathrm{CHO})_{2}$ & ${ }^{1} A_{\mathrm{u}}$ & 2.72 & 2.20 & 2.18 & 2.16 & 2.08 & 2.06 \\
\hline Benzene & ${ }^{1} B_{2 \mathrm{u}}$ & 4.72 & 4.92 & 4.91 & 4.91 & 4.92 & 4.92 \\
\hline Pyridine & ${ }^{1} B_{2}$ & 4.76 & $5.02^{\mathrm{a}}$ & $5.02^{\mathrm{a}}$ & $5.01^{\mathrm{a}}$ & $5.01^{\mathrm{a}}$ & $5.00^{\mathrm{a}}$ \\
\hline Porphyrin & ${ }^{1} B_{1 \mathrm{u}}$ & $(2.02)$ & $(1.89)$ & (1.90) & $(1.89)$ & (1.91) & $(1.92)$ \\
\hline RMS (S) & & & 0.42 & 0.42 & 0.42 & 0.46 & 0.45 \\
\hline MAX (S) & & & 0.70 & 0.70 & 0.70 & 0.75 & 0.75 \\
\hline RMS (T) & & & 0.74 & 0.74 & 0.74 & 0.63 & 0.64 \\
\hline $\operatorname{MAX}(\mathrm{T})$ & & & 1.59 & 1.59 & 1.59 & 1.28 & 1.28 \\
\hline RMS & & & 0.57 & 0.57 & 0.57 & 0.53 & 0.53 \\
\hline MAX & & & 1.59 & 1.59 & 1.59 & 1.28 & 1.28 \\
\hline
\end{tabular}

${ }^{\mathrm{a}}$ Saddle point.

difference between two hole orbitals. In such case, excitation energies are largely described with the DFTB1 level, so the difference between excitation energies calculated with DFTB2 and DFTB3 is small.

TD-DFTB3/3OB nevertheless improved the prediction of adiabatic excitation energies slightly, by comparing RMS and maximum deviations for all items at the bottom in Table II. The improvement is largely attributed to the excitation energies for CO molecule. Since the extension to TD-DFTB3 itself does not improve excitation energies dramatically, the small improvement comes from the difference of two parameter sets, $\mathrm{MIO}$ and 3OB. In fact, TD-DFTB2/3OB gives an adiabatic excitation energy of $7.32 \mathrm{eV}$ for $\mathrm{CO}$, so the excitation energy for $\mathrm{CO}$ is dominantly controlled by the employed parameter set. The excitation corresponds to the HOMO-LUMO $\left(\pi-\pi^{*}\right)$ transition (both HOMO and LUMO are doubly generated), and the HOMO-LUMO gaps of the optimized CO geometry on the excited potential energy surface (PES) with TD-DFTB2 and TD-DFTB3 are 6.66 and $6.38 \mathrm{eV}$, respectively, so the smaller adiabatic excitation energy of TD-DFTB3 is attributed to the difference of the HOMO-LUMO gap. It should be noted that the $3 \mathrm{OB}$ parameter set uses more diffuse basis functions in parameterization than those for the older MIO parameter set so as to describe weak interactions correctly. ${ }^{17}$

Another remarkable difference is found for the adiabatic excitation energy for $\mathrm{CS}_{2}$ molecule; the deviation of $0.46 \mathrm{eV}$ with TD-DFTB2/MIO is decreased to $0.02 \mathrm{eV}$ with TDDFTB3/3OB. The excitation is characterized as a single orbital transition from HOMO-1 $\left(A_{2}\right)$ to LUMO $\left(A_{1}\right)$. For the optimized geometry at $\mathrm{S}_{2}$, an interesting difference is obvious for LUMOs in Figure 1, visualized with VMD (Visual Molecular Dynamics). ${ }^{51}$ DFTB2/MIO shows $\pi^{*}$ character orbitals between $\mathrm{C}$ and S, while DFTB3/3OB does $\pi$ orbitals. The difference of LUMOs should be attributed to the different levels of non-interacting d orbitals in two parameters. After optimizing parameters for DFTB $3,{ }^{17}$ the level of $\mathrm{d}$ orbitals for $3 \mathrm{OB}$ is higher than that for $\mathrm{MIO}$ by 0.3 hartree, and this reduces the participation of $\mathrm{d}$ orbitals in MO hybridization, which is supposed to be good for describing $\mathrm{O}-\mathrm{S}$ bonds, while less good for $\mathrm{C}-\mathrm{S}$. As a reference, we optimized $\mathrm{CS}_{2}$ with TD-PBE/STO$3 \mathrm{G}$ whose gradients are obtained through numerical differentiations at $\mathrm{S}_{2}\left({ }^{3} A_{2}\right)$. The STO-3G basis set was employed in accordance with the fact that DFTB uses minimal orbitals. We performed a TD-PBE/6-311+G* calculation and verified that the character of the excitation with STO-3G and $6-311+\mathrm{G}^{*}$ is very similar. The character of excitation of TD-PBE/STO-3G

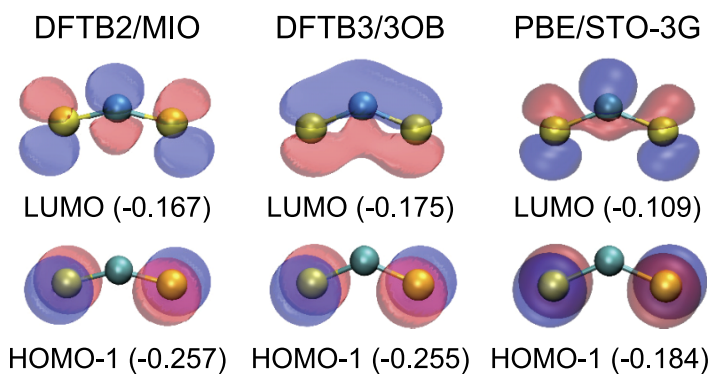

FIG. 1. HOMOs-1 and LUMOs $\left( \pm 0.05\left(e / a_{0}\right)^{1 / 2}\right.$ isosurface $)$ of the optimized $\mathrm{CS}_{2}$ with TD-DFTB2/MIO, TD-DFTB3/3OB, and TD-PBE/STO-3G at the ${ }^{3} A_{2}$ excited state. Values in parentheses are the eigenvalues of vectors (unit in hartree). 
TABLE III. RMS and maximum (MAX) deviations for bond distance (unit in $\AA$ ) and bond and dihedral angles (degree) with respect to experiment for molecules in Table II.

\begin{tabular}{|c|c|c|c|c|c|}
\hline & TD-DFTB2/MIO & TD-DFTB3/MIO/calc & TD-DFTB3/MIO/fit & TD-DFTB2/3OB & TD-DFTB3/3OB \\
\hline MAX (distance) & 0.053 & 0.054 & 0.054 & 0.048 & 0.048 \\
\hline MAX (angle) & 15.05 & 15.17 & 15.21 & 17.40 & 17.27 \\
\hline RMS (dihedral) & 16.65 & 17.48 & 17.45 & 20.67 & 22.05 \\
\hline MAX (dihedral) & 19.29 & 19.73 & 19.73 & 21.48 & 22.32 \\
\hline
\end{tabular}

is very similar to that of TD-DFTB too, and the HOMO-1 and LUMO calculated with PBE/STO-3G are quite similar with those of DFTB2/MIO, but the LUMO is very different from the one obtained with DFTB3/3OB. It makes us to conclude that the good agreement for $\mathrm{CS}_{2}$ with TD-DFTB3/3OB may come from a fortunate error cancellation. Since the choice of the level of $d$ orbitals in Ref. 17 is originated by better agreement for $\mathrm{O}-\mathrm{S}$ species, the parameters for $\mathrm{S}$ and $\mathrm{P}$ are not optimal for excited state calculations.

As for $\mathrm{CH}_{2} \mathrm{~S}$, the excitation with TD-DFTB2/MIO is described by a pure HOMO-LUMO transition, so the difference between singlet-singlet and singlet-triplet excitation energies is exactly zero, which is consistent with the previous observation..$^{21}$ Moreover, the symmetry of the stationary points at ${ }^{1} A_{2}$ and ${ }^{3} A^{\prime \prime}$ is supposed to be $C_{2 \mathrm{v}}$ and $C_{\mathrm{s}}$, but the optimized geometries with the MIO parameter set belong to $C_{2 \mathrm{v}}$ in both cases. The ${ }^{3} A^{\prime \prime}$ state is supposed to give two hydrogen atoms out-of-plane, but we could not observe such a geometry with the MIO parameter set. Interestingly, TD-DFTB2/3OB and TD-DFTB3/3OB give two out-of-plane hydrogen atoms at the ${ }^{3} A^{\prime \prime}$ state. Actually, ${ }^{1} A_{2}$ also gives out-of-plane hydrogen atoms if geometry optimization is started with out-of-plane hydrogen atoms. The reason of the difference should be attributed to the difference of MO hybridization with the MIO and $3 \mathrm{OB}$ parameters too.

Since we can compute oscillator strengths with TD-DFTB using dipole integrals, we compare those of TD-DFTB and a set of reference calculations with TD-PBE/STO-3G. Among 11 singlet-singlet excitations, TD-PBE/STO-3G gives nontrivial oscillator strengths for $\mathrm{CO}(0.018), \mathrm{HCN}(0.004), \mathrm{C}_{2} \mathrm{H}_{2}$ (0.005), and pyridine (0.001), while TD-DFTB gives nontrivial oscillator strengths only for $\mathrm{CH}_{2} \mathrm{O}(0.002$ and 0.003 with $\mathrm{MIO}$ and $3 \mathrm{OB}$ parameter sets). The discrepancy comes from two reasons. Zero oscillator strengths in TD-DFTB for $\mathrm{CO}, \mathrm{HCN}, \mathrm{C}_{2} \mathrm{H}_{2}$, and pyridine are attributed to the lack of on-site interactions. ${ }^{52}$ On the other hand, the non-trivial oscillator strengths for $\mathrm{CH}_{2} \mathrm{O}$ come from a difference in electronic structure between DFTB and PBE/STO-3G. Note that the characters of excitation for $\mathrm{CH}_{2} \mathrm{O}$ with TD-DFTB and TD-PBE/STO-3G are nevertheless same.

As experimental geometrical parameters (bond distance and bond and dihedral angles) for molecules in Table II are available, ${ }^{9}$ we tested the five DFTB methods by comparing these parameters. A similar comparison for TD-DFTB2/MIO has been done in Ref. 21. The geometrical parameters are overall better predicted with the MIO parameter set, although the difference between the predictions with the $\mathrm{MIO}$ and $3 \mathrm{OB}$ parameter sets is rather small (Table III).

\section{B. Adiabatic excitation energies for medium-size dye molecules}

Considering the efficiency of TD-DFTB as shown in Table I, it is possible to predict adiabatic excitation energies of bigger molecules with TD-DFTB relatively easily. Goerigk et al. proposed a benchmark set consisting of 12 dye molecules in their works. ${ }^{49,50}$ In order to check the applicability of TD-DFTB further, we have calculated adiabatic excitation energies for 1-3 and 5-12 (see Ref. 50 for structures) in vacuum with five TD-DFTB methods. For $\mathbf{4}$, we do not have appropriate parameters. Calculated and reference TD-PBE/TZVP ${ }^{49,50}$ energies as well as RMS and maximum deviations with respect to TD-PBE/TZVP are summarized in Table IV. Additional set of parameters for halogen atoms ${ }^{37,53}$ were utilized.

With the MIO parameter set, we could not obtain excited state minima for $\mathbf{1}$ and $\mathbf{1 2}$. These molecules contain halogen atoms, $\mathrm{F}$ in $\mathbf{1}$ and $\mathrm{Cl}$ in 12. Particularly, in 1, the optimized geometry with the MIO parameter set at the ground state gives $\mathrm{a}-\mathrm{CF}_{3}$ group in which all four atoms in the group are on a same plane. Such a situation is totally inconsistent with our intuition, so we guess that the artefact should be attributed to the limit of the parameters. We did not face with such a difficulty with the 3OB parameter set. For fair comparison, $\mathbf{1}$ and $\mathbf{1 2}$ are excluded in computing RMS and maximum deviations.

The RMS and maximum deviations in Table IV indicate that the $3 \mathrm{OB}$ parameter set improves the prediction of adiabatic excitation energies slightly. Among five TD-DFTB methods, TD-DFTB3/3OB gives the smallest deviation from the reference calculation, but the improvement over TDDFTB2/3OB is little. In computing adiabatic excitation energies, the semi-numerical second derivative with analytical gradient is certainly the most time consuming step. With TDDFTB for the biggest molecule (5; 60 atoms), it can be finished in around 2 min using one CPU core of Xeon E5-1620 v3 $(3.50 \mathrm{GHz})$.

\section{Absorption and fluorescence energies of cresyl violet}

In the end, we demonstrate one possible application of TD-DFTB. Taking the fact that DFTB3 is supposed to be superior to DFTB2 for polar and hydrogen bonded systems, it is still early to conclude that TD-DFTB3 does not improve excited state properties. We therefore calculated the absorption and fluorescence energies of cresyl violet, ${ }^{54}$ which contains one positive charge, in explicit water molecules as a practical 
TABLE IV. Adiabatic excitation energies (unit in eV) for 11 dye molecules, obtained with TD-PBE/TZVP ${ }^{49,50}$ and five TD-DFTB methods. RMS and maximum (MAX) deviations (unit in $\mathrm{eV}$ ) for $\mathbf{2 , 3}$, and 5-11 with respect to TD-PBE/TZVP are shown at the bottom.

\begin{tabular}{|c|c|c|c|c|c|c|}
\hline & TD-PBE/TZVP & TD-DFTB2/MIO & TD-DFTB3/MIO/calc & TD-DFTB3/MIO/fit & TD-DFTB2/3OB & TD-DFTB3/3OB \\
\hline 2 & 2.13 & 2.20 & 2.20 & 2.20 & 2.23 & 2.23 \\
\hline 5 & 1.86 & $2.06^{\mathrm{b}}$ & $2.05^{\mathrm{b}}$ & $2.04^{\mathrm{b}}$ & $2.04^{\mathrm{b}}$ & $2.04^{\mathrm{b}}$ \\
\hline 6 & 2.22 & 2.30 & 2.30 & 2.30 & 2.30 & 2.30 \\
\hline 7 & 3.05 & 2.82 & $2.80^{\mathrm{b}}$ & 2.79 & 3.05 & 3.00 \\
\hline 10 & 2.33 & 2.26 & 2.24 & 2.23 & 2.28 & 2.25 \\
\hline 11 & 1.81 & 1.82 & 1.81 & 1.81 & 1.81 & 1.80 \\
\hline 12 & 1.48 & $\ldots^{\mathrm{a}}$ & $\ldots^{\mathrm{a}}$ & $\ldots^{\mathrm{a}}$ & 1.91 & 1.82 \\
\hline RMS & & 0.13 & 0.13 & 0.13 & 0.10 & 0.10 \\
\hline MAX & & 0.23 & 0.25 & 0.26 & 0.18 & 0.17 \\
\hline
\end{tabular}

${ }^{\mathrm{a}}$ Unable to locate excited state minimum.

${ }^{\mathrm{b}}$ Saddle point.

test example. We soaked cresyl violet at 10 locations of a large water box optimized with the "minimize" module in TINKER $6.0^{55}$ with the AMBER99 force field parameters. ${ }^{56}$ Surrounding water molecules were then removed other than those located at $2.0<d<6.0$, where $d$ is the distance between the solute (cresyl violet) atoms and water molecules in $\AA$. The resultant geometries contained 323-404 atoms (cresyl violet plus 97 to 124 water molecules). They were optimized with (TD-)DFTB2/MIO and (TD-)DFTB3/3OB, respectively, and excitation energies were calculated for the optimized structures at the ground state $\left(\mathrm{S}_{0}\right)$ for absorption and at the first excited state $\left(S_{1}\right)$ for fluorescence energies. With explicit water molecules, excited state geometry optimizations were started with the optimized structure at the ground state with the corresponding DFTB method, but we sometimes had to start with the TD-DFTB3 structures in some TD-DFTB2 calculations because of technical problems. We added the UFF (universal force field)-type dispersion correction ${ }^{57,58}$ in all calculations here. Calculated (in vacuum and explicit water) and experimental ${ }^{54}$ (in water) absorption and fluorescence energies and oscillator strengths are summarized in Table V.

The excitation mainly corresponds to the transition from HOMO to LUMO. These orbitals with DFTB3 for one of the optimized geometries on $\mathrm{S}_{1}$ PES in vacuum and water are depicted in Figure 2. The absorption energies in vacuum and water do not differ significantly, but the fluorescence energies are different by around $0.2 \mathrm{eV}$. This clearly indicates the importance of solvent effects. The experimental fluorescence energy $^{54}$ in water is $1.96 \mathrm{eV}(631 \mathrm{~nm})$, which is very well reproduced by our TD-DFTB3 calculation as well as TDDFTB2. Of course, a part of the good agreement may be attributed to a fortunate error cancellation and an arbitrariness of sampling, but it is quite probable that the improvement of (TD-)DFTB3 in describing polar and hydrogen bonded systems is important for the prediction of fluorescence energies.

The difference of fluorescence energies with TD-DFTB2 and TD-DFTB3 is only $0.03 \mathrm{eV}$. Although it implies that the difference between TD-DFTB2 and TD-DFTB3 methods is trivial again, we would like to point out that the geometry optimization on the excited state PES with TD-DFTB2 was rather difficult, because the order of excited state easily alters during geometry optimization. Actually, we could not directly find stationary points on $\mathrm{S}_{1}$ PES from the $\mathrm{S}_{0}$ optimized structure with DFTB2 as mentioned. Some additional calculations prove that such a difficulty caused by state alternation is suppressed by adding only the third-order contributions of a Taylor series of the DFT energy, but not by the damping function for hydrogen atoms. This indicates that the DFTB3 contributions improve the description of excited state PESs, but we need more analysis to conclude it.

According to oscillator strengths with TD-DFTB3 (Table V), transition intensity is very slightly (3\%) enhanced upon solvation, which is consistent with a study for a

TABLE V. Experimental and calculated absorption and fluorescence energies (unit in eV) for cresyl violet and oscillator strengths. Calculated energies and oscillator strengths in water are the averages of 10 geometries. Values in parentheses represent lower and upper bounds of 10 energies.

\begin{tabular}{lccccc}
\hline \hline \multirow{2}{*}{ Method } & \multicolumn{2}{c}{ Excitation energies } & & \multicolumn{2}{c}{ Oscillator strength } \\
\cline { 2 - 3 } & Absorption & Fluorescence & & Absorption & Fluorescence \\
\hline TD-DFTB2 (in vacuum) & 2.46 & 2.10 & & 0.452 & 0.200 \\
TD-DFTB3 (in vacuum) & 2.50 & 2.15 & & 0.429 & 0.187 \\
TD-DFTB2 (in water) & $2.40(2.35 / 2.44)$ & $1.93(1.80 / 2.03)$ & & 0.427 & 0.175 \\
TD-DFTB3 (in water) & $2.46(2.43 / 2.49)$ & $1.96(1.86 / 2.09)$ & & 0.442 & 0.157 \\
Experiment (in water) & 2.12 & 1.96 & & N/A & N/A \\
\hline \hline
\end{tabular}




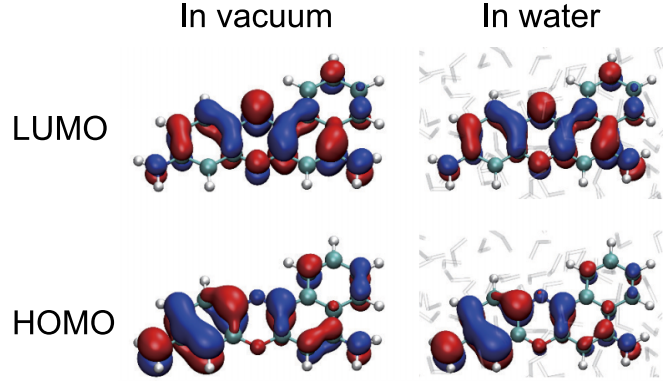

FIG. 2. HOMOs and LUMOs $\left( \pm 0.03\left(e / a_{0}\right)^{1 / 2}\right.$ isosurface $)$ of the optimized cresyl violet in vacuum and water with TD-DFTB3/3OB on $\mathrm{S}_{1}$ PES.

explicitly hydrated acetone system. ${ }^{59}$ On the other hand, the transition intensity is diminished with TD-DFTB2. The oscillator strengths for fluorescence are diminished in both TD-DFTB2 and TD-DFTB3 in solution phase. A direct comparison between the results of the present study and those of Ref. 59 is complicated, owing to the different characters of the excitations involved.

\section{CONCLUSIONS}

We derived the time-dependent density-functional tightbinding method with the third-order contributions of a Taylor series expansion of the DFT energy (TD-DFTB3). The developed method was implemented in GAMESSUS. The analytical gradient requires to compute the thirdorder derivative of the total energy with respect to density matrix elements, while it disappears for TD-DFTB2. ${ }^{21}$ The computational cost to include DFTB3 contributions for excitation energies is virtually negligible.

Since the performance of TD-DFTB3 has been completely unknown, we compared a set of adiabatic excitation energies with TD-DFTB2 and TD-DFTB3. We showed that the inclusion of the DFTB3 terms has small effects for excitation energies, while the different parameter sets optimized for DFTB3 slightly improved the prediction of adiabatic excitation energies. The small difference of excitation energies comes from the fact that the third-order contributions affect orbital energies very little. Of course, one has to realize that DFTB3 is developed to describe charge polarized and hydrogen bonded systems better, but not excited state properties. Although the parameters optimized for DFTB3 slightly improved the prediction of excitation energies, the improvement could be partly attributed to a fortunate error cancellation. We expect that the rangeseparated version of DFTB ${ }^{60}$ will improve excitation energies, as that of DFT did improve well. ${ }^{10}$

We also demonstrated a possible application of TDDFTB with cresyl violet solvated by explicit water molecules. Although the absorption energies were overestimated relative to the experimental result, ${ }^{54}$ the agreement of the TD-DFTB3 fluorescence energy with the experiment was fairly promising. We pointed out that DFTB3 contributions may improve the description of excited state PESs. Since many parameters are being produced for bio-systems with DFTB3, we think it is important to shift from DFTB2 to DFTB3 for predicting excited state properties, too. At present, solvent effects are taken into account with explicit molecules, and this could introduce the arbitrariness of positions and orientations of solvent molecules. To eliminate such an arbitrariness, the implicit treatment ${ }^{61}$ is of great interest as a future work. We are also planning to apply TD-DFTB2 and TD-DFTB3 for the fragment molecular orbital-based DFTB. ${ }^{62,63}$

\section{ACKNOWLEDGMENTS}

Y.N. acknowledges the Fukui Institute for Fundamental Chemistry Fellowship. The author would like to thank Dr. Dmitri G. Fedorov and Mr. Kosuke Usui for helpful comments.

\section{APPENDIX A: DERIVATION OF Z-VECTOR EQUATIONS FOR TD-DFTB3}

The derivative of the auxiliary Lagrangian functional in Eq. (27) is

$$
\begin{aligned}
\frac{\partial L}{\partial C_{\mu p \sigma}}= & \frac{\partial G[\mathbf{X}, \mathbf{Y}, \omega]}{\partial C_{\mu p \sigma}}+\sum_{i a \tau} Z_{i a \tau} \frac{\partial H_{i a \tau}}{\partial C_{\mu p \sigma}} \\
& -\sum_{r s \tau, r \leq s} W_{r s \tau} \frac{\partial S_{r s \tau}}{\partial C_{\mu p \sigma}},
\end{aligned}
$$

which is equal to zero, and solving Z-vector equations corresponds to finding the Lagrangian multipliers $\mathbf{Z}$ and $\mathbf{W}$. Because a straightforward derivative is hard to manipulate equations, one usually multiplies both sides of the equation with $C_{\mu q \sigma}$ and sums over $\mu$, giving

$$
\begin{aligned}
Q_{p q \sigma} & +\sum_{i a \tau} Z_{i a \tau} \sum_{\mu} \frac{\partial H_{i a \tau}}{\partial C_{\mu p \sigma}} C_{\mu q \sigma} \\
= & \sum_{r s \tau, r \leq s} W_{r s \tau} \sum_{\mu} \frac{\partial S_{r s \tau}}{\partial C_{\mu p \sigma}} C_{\mu q \sigma},
\end{aligned}
$$

where

$$
Q_{p q \sigma}=\sum_{\mu} \frac{\partial G[\mathbf{X}, \mathbf{Y}, \omega]}{\partial C_{\mu p \sigma}} C_{\mu q \sigma} .
$$

Since $p$ and $q$ label general MOs, they could be occupied and virtual orbitals. Considering that we do not need the derivative of density matrix elements when $p$ belongs to virtual orbitals and using the diagonal nature of the Hamiltonian matrix in MO basis, we can express $Q_{p q \sigma}$ matrix decomposing into four blocks: occupied-occupied ( $p=i \leq q=j$ ), occupied-virtual $(p=i$ and $q=a)$, virtual-occupied $(p=a$ and $q=i)$, and virtual-virtual ( $p=a \leq q=b)$ blocks,

$$
\begin{aligned}
Q_{i j \sigma}= & \omega \sum_{a}\left\{(X+Y)_{i a \sigma}(X-Y)_{j a \sigma}\right. \\
& \left.+(X-Y)_{i a \sigma}(X+Y)_{j a \sigma}\right\} \\
& -\sum_{a} \varepsilon_{a \sigma}\left\{(X+Y)_{i a \sigma}(X+Y)_{j a \sigma}\right. \\
& \left.+(X-Y)_{i a \sigma}(X-Y)_{j a \sigma}\right\} \\
& +H_{i j \sigma}^{+}[\mathbf{T}] \\
& +2 \sum_{k c \tau l d v} \hat{g}_{i j \sigma, k c \tau, l d v}(X+Y)_{k c \tau}(X+Y)_{l d v},
\end{aligned}
$$




$$
\begin{aligned}
Q_{i a \sigma}= & \sum_{b}(X+Y)_{i b \sigma} H_{a b \sigma}^{+}[(\mathbf{X}+\mathbf{Y})]+H_{i a \sigma}^{+}[\mathbf{T}] \\
& +2 \sum_{j b \tau k c v} \hat{g}_{i a \sigma, j b \tau, k c v}(X+Y)_{j b \tau}(X+Y)_{k c v} \\
& Q_{a i \sigma}=\sum_{j}(X+Y)_{j a \sigma} H_{j i \sigma}^{+}[(\mathbf{X}+\mathbf{Y})]
\end{aligned}
$$

and

$$
\begin{aligned}
Q_{a b \sigma}= & \omega \sum_{i}\left\{(X+Y)_{i a \sigma}(X-Y)_{i b \sigma}\right. \\
& \left.+(X-Y)_{i a \sigma}(X+Y)_{i b \sigma}\right\} \\
& -\sum_{i} \varepsilon_{i \sigma}\left\{(X+Y)_{i a \sigma}(X+Y)_{i b \sigma}\right. \\
& \left.+(X-Y)_{i a \sigma}(X-Y)_{i b \sigma}\right\},
\end{aligned}
$$

where the unrelaxed difference density matrix $\mathbf{T}$ is

$$
\begin{aligned}
T_{i j \sigma}= & -\frac{1}{2} \sum_{a}\left\{(X+Y)_{i a \sigma}(X+Y)_{j a \sigma}\right. \\
& \left.+(X-Y)_{i a \sigma}(X-Y)_{j a \sigma}\right\}, \\
T_{i a \sigma}= & 0, \\
T_{a i \sigma}= & 0, \\
T_{a b \sigma}= & \frac{1}{2} \sum_{i}\left\{(X+Y)_{i a \sigma}(X+Y)_{i b \sigma}\right. \\
& \left.+(X-Y)_{i a \sigma}(X-Y)_{i b \sigma}\right\} .
\end{aligned}
$$

The derivative of the Hamiltonian and overlap matrices is similarly obtained,

$$
\begin{aligned}
\sum_{i a \tau} Z_{i a \tau} \sum_{\mu} \frac{\partial H_{i a \tau}}{\partial C_{\mu p \sigma}} C_{\mu q \sigma}= & \sum_{i a \tau} \delta_{\sigma \tau}\left(\delta_{i p} \delta_{q a}+\delta_{a p} \delta_{i q}\right) \varepsilon_{q \sigma} Z_{i a \tau} \\
& +\sum_{j} \delta_{j p} H_{p q \sigma}^{+}[\mathbf{Z}]
\end{aligned}
$$

and

$$
\sum_{r s \tau, r \leq s} W_{r s \tau} \sum_{\mu} \frac{\partial S_{r s \tau}}{\partial C_{\mu p \sigma}} C_{\mu q \sigma}=\left(1+\delta_{p q}\right) W_{p q \sigma} .
$$

Now, by combining equations above, we can obtain a set of linear equations for different blocks,

$$
\begin{aligned}
& Q_{i j \sigma}+H_{i j \sigma}^{+}[\mathbf{Z}]=\left(1+\delta_{i j}\right) W_{i j \sigma}, \\
& Q_{i a \sigma}+\varepsilon_{a \sigma} Z_{i a \sigma}+H_{i a \sigma}^{+}[\mathbf{Z}]=W_{i a \sigma}, \\
& Q_{a i \sigma}+\varepsilon_{i \sigma} Z_{i a \sigma}=W_{i a \sigma}, \\
& Q_{a b \sigma}=\left(1+\delta_{a b}\right) W_{a b \sigma} .
\end{aligned}
$$

Subtracting Eqs. (A12) and (A13), one obtains a Z-vector equation,

$\left(\varepsilon_{a \sigma}-\varepsilon_{i \sigma}\right) Z_{i a \sigma}+H_{i a \sigma}^{+}[\mathbf{Z}]=-\left(Q_{i a \sigma}-Q_{a i \sigma}\right)$,

which is equivalent to Eq. (29). Once we obtain the vector $\mathbf{Z}$ as a solution of a Z-vector equation, we can then evaluate the vector $\mathbf{W}$, according to

$$
\begin{aligned}
W_{i j \sigma} & =\frac{1}{1+\delta_{i j}}\left(Q_{i j \sigma}+H_{i j \sigma}^{+}[\mathbf{Z}]\right), \\
W_{a b \sigma} & =\frac{1}{1+\delta_{a b}} Q_{a b \sigma}, \\
W_{i a \sigma} & =Q_{a i \sigma}+\varepsilon_{i \sigma} Z_{i a \sigma} .
\end{aligned}
$$

\section{APPENDIX B: EXPLICIT EXCITATION ENERGY GRADIENT FOR TD-DFTB3}

According to Eq. (36), after mathematical manipulations, the explicit excitation energy gradient for singlet-singlet excitation is

$$
\begin{aligned}
\frac{\partial \omega}{\partial R_{\alpha x}}= & 2 \sum_{\sigma} \sum_{A \neq \alpha} \sum_{\mu \in A} \sum_{\nu \in \alpha}\left\{P_{\mu \nu \sigma}\left(\frac{\partial H_{\mu \nu}^{0}}{\partial R_{\alpha x}}+\frac{\partial S_{\mu v}}{\partial R_{\alpha x}} \Omega_{A B}\right)\right. \\
& -W_{\mu \nu \sigma} \frac{\partial S_{\mu \nu}}{\partial a}+D_{\mu \nu \sigma} \frac{\partial S_{\mu \nu}}{\partial a} \Omega_{A B}^{P, X Y, \mathrm{~S}} \\
& \left.+2(X+Y)_{\mu \nu \sigma} \frac{\partial S_{\mu v}}{\partial R_{\alpha x}} \Omega_{A B}^{X Y, \mathrm{~S}}\right\} \\
& +2 \sum_{B \neq \alpha} q_{\alpha}^{X Y} q_{B}^{X Y} \frac{\partial \gamma_{A \alpha}}{\partial R_{\alpha x}} \\
& +\frac{2}{3} \sum_{B \neq \alpha}\left\{2 q_{\alpha}^{X Y} q_{B}^{X Y}\left(\Delta q_{\alpha} \frac{\partial \Gamma_{\alpha B}}{\partial R_{\alpha x}}+\Delta q_{B} \frac{\partial \Gamma_{B \alpha}}{\partial R_{\alpha x}}\right)\right. \\
& \left.+\left(q_{\alpha}^{X Y}\right)^{2} \Delta q_{B} \frac{\partial \Gamma_{\alpha B}}{\partial R_{\alpha x}}+\left(q_{B}^{X Y}\right)^{2} \Delta q_{\alpha} \frac{\partial \Gamma_{B \alpha}}{\partial R_{\alpha x}}\right\} \\
& +\sum_{B \neq \alpha}\left(q_{\alpha}^{P} \Delta q_{B}+\Delta q_{\alpha} q_{B}^{P}\right) \frac{\partial \gamma_{\alpha B}}{\partial R_{\alpha x}} \\
& +\frac{1}{3} \sum_{B \neq \alpha}\left\{2 \Delta q_{\alpha} \Delta q_{B}\left(q_{\alpha}^{P} \frac{\partial \Gamma_{\alpha B}}{\partial R_{\alpha x}}+q_{B}^{P} \frac{\partial \Gamma_{B \alpha}}{\partial R_{\alpha x}}\right)\right. \\
& \left.+\left(\Delta q_{\alpha}\right)^{2} q_{B}^{P} \frac{\partial \Gamma_{\alpha B}}{\partial R_{\alpha x}}+\left(\Delta q_{B}\right)^{2} q_{\alpha}^{P} \frac{\partial \Gamma_{B \alpha}}{\partial R_{\alpha x}}\right\}
\end{aligned}
$$

where

$$
\begin{aligned}
q_{A}^{P} & =\sum_{\sigma} \sum_{\mu \in A} \sum_{\nu} P_{\mu \nu \sigma} S_{\mu \nu}, \\
\Omega_{A B}^{P, X Y, \mathrm{~S}}= & \frac{1}{2} \sum_{C}\left(\gamma_{A C}+\gamma_{B C}\right) q_{C}^{P} \\
& +\frac{1}{3} \sum_{C}\left\{\left(\Gamma_{A C} \Delta q_{A}+\Gamma_{B C} \Delta q_{B}\right) q_{C}^{P}\right. \\
& +\left(\Gamma_{A C} q_{A}^{P}+\Gamma_{B C} q_{B}^{P}\right) \Delta q_{C} \\
& \left.+\left(\Gamma_{C A}+\Gamma_{C B}\right) q_{C}^{P} \Delta q_{C}\right\} \\
& +\frac{1}{3} \sum_{C}\left\{2\left(\Gamma_{A C} q_{A}^{X Y}+\Gamma_{B C} q_{B}^{X Y}\right) q_{C}^{X Y}\right. \\
& \left.+\left(\Gamma_{C A}+\Gamma_{C B}\right)\left(q_{C}^{X Y}\right)^{2}\right\},
\end{aligned}
$$

and

$$
\begin{aligned}
\Omega_{A B}^{X Y, \mathrm{~S}}= & \frac{1}{2} \sum_{C}\left(\gamma_{A C}+\gamma_{B C}\right) q_{C}^{X Y} \\
& +\frac{1}{3} \sum_{C}\left\{\left(\Gamma_{A C} \Delta q_{A}+\Gamma_{B C} \Delta q_{B}\right) q_{C}^{X Y}\right. \\
& +\left(\Gamma_{A C} q_{A}^{X Y}+\Gamma_{B C} q_{B}^{X Y}\right) \Delta q_{C} \\
& \left.+\left(\Gamma_{C A}+\Gamma_{C B}\right) q_{C}^{X Y} \Delta q_{C}\right\} .
\end{aligned}
$$

For singlet-triplet excitation,

$$
\frac{\partial \omega}{\partial R_{\alpha x}}=2 \sum_{\sigma} \sum_{A \neq \alpha} \sum_{\mu \in A} \sum_{\nu \in \alpha}\left\{P_{\mu \nu \sigma}\left(\frac{\partial H_{\mu \nu}^{0}}{\partial R_{\alpha x}}+\frac{\partial S_{\mu \nu}}{\partial R_{\alpha x}} \Omega_{A B}\right)\right.
$$




$$
\begin{aligned}
& -W_{\mu \nu \sigma} \frac{\partial S_{\mu \nu}}{\partial a}+D_{\mu \nu \sigma} \frac{\partial S_{\mu \nu}}{\partial a} \Omega_{A B}^{P, \mathrm{~T}} \\
& \left.+2(X+Y)_{\mu \nu \sigma} \frac{\partial S_{\mu \nu}}{\partial R_{\alpha x}} \Omega_{A B}^{X Y, \mathrm{~T}}\right\} \\
& +2 \sum_{B \neq \alpha} q_{\alpha}^{X Y} q_{B}^{X Y} \frac{\partial \gamma_{A \alpha}}{\partial R_{\alpha x}} \\
& +\sum_{B \neq \alpha}\left(q_{\alpha}^{P} \Delta q_{B}+\Delta q_{\alpha} q_{B}^{P}\right) \frac{\partial \gamma_{\alpha B}}{\partial R_{\alpha x}} \\
& +\frac{1}{3} \sum_{B \neq \alpha}\left\{2 \Delta q_{\alpha} \Delta q_{B}\left(q_{\alpha}^{P} \frac{\partial \Gamma_{\alpha B}}{\partial R_{\alpha x}}+q_{B}^{P} \frac{\partial \Gamma_{B \alpha}}{\partial R_{\alpha x}}\right)\right. \\
& \left.+\left(\Delta q_{\alpha}\right)^{2} q_{B}^{P} \frac{\partial \Gamma_{\alpha B}}{\partial R_{\alpha x}}+\left(\Delta q_{B}\right)^{2} q_{\alpha}^{P} \frac{\partial \Gamma_{B \alpha}}{\partial R_{\alpha x}}\right\}
\end{aligned}
$$

where

$$
\begin{aligned}
\Omega_{A B}^{P, \mathrm{~T}}= & \frac{1}{2} \sum_{C}\left(\gamma_{A C}+\gamma_{B C}\right) q_{C}^{P} \\
& +\frac{1}{3} \sum_{C}\left\{\left(\Gamma_{A C} \Delta q_{A}+\Gamma_{B C} \Delta q_{B}\right) q_{C}^{P}\right. \\
& +\left(\Gamma_{A C} q_{A}^{P}+\Gamma_{B C} q_{B}^{P}\right) \Delta q_{C} \\
& \left.+\left(\Gamma_{C A}+\Gamma_{C B}\right) q_{C}^{P} \Delta q_{C}\right\}
\end{aligned}
$$

and

$$
\begin{aligned}
\Omega_{A B}^{X Y, \mathrm{~T}}= & \frac{1}{2} \sum_{C}\left(\gamma_{A C}+\gamma_{B C}\right) q_{C}^{X Y} \\
& +\frac{1}{2}\left(M_{A} q_{A}^{X Y}+M_{B} q_{B}^{X Y}\right) .
\end{aligned}
$$

By omitting the $\Gamma$-related terms, one obtains TD-DFTB2 gradient. The gradient expressions for excitation energies (Eqs. (B1) and (B5)) resemble to that for the ground state (Eq. (9)). These gradients can therefore be calculated simultaneously.

${ }^{1}$ E. Runge and E. K. U. Gross, Phys. Rev. Lett. 52, 997 (1984).

${ }^{2}$ M. E. Casida, "Time-dependent density functional response theory for molecules," in Recent Advances in Density Functional Methods, edited by D. P. Chong (World Scientific, Singapore, 1995), Chap. 5, pp. 155-192.

${ }^{3}$ F. Furche, J. Chem. Phys. 114, 5982 (2001).

${ }^{4}$ A. Dreuw and M. Head-Gordon, Chem. Rev. 105, 4009 (2005).

${ }^{5}$ M. E. Casida, J. Mol. Struct.: THEOCHEM 914, 3 (2009).

${ }^{6}$ Fundamentals of Time-Dependent Density Functional Theory, edited by M. A. L. Marques, N. T. Maitra, F. M. S. Nogueira, E. K. U. Gross, and A. Rubio (Springer Berlin Heidelberg, Berlin, 2012).

${ }^{7}$ H. Weiss, R. Ahlrichs, and M. Häser, J. Chem. Phys. 99, 1262 (1993).

${ }^{8}$ R. E. Stratmann, G. E. Scuseria, and M. J. Frisch, J. Chem. Phys. 109, 8218 (1998).

${ }^{9}$ F. Furche and R. Ahlrichs, J. Chem. Phys. 117, 7433 (2002).

${ }^{10}$ M. Chiba, T. Tsuneda, and K. Hirao, J. Chem. Phys. 124, 144106 (2006).

${ }^{11}$ G. Scalmani, M. J. Frisch, B. Mennucci, J. Tomasi, R. Cammi, and V. Barone, J. Chem. Phys. 124, 094107 (2006)

${ }^{12}$ J. Liu and W. Liang, J. Chem. Phys. 135, 014113 (2011).

${ }^{13}$ J. Liu and W. Liang, J. Chem. Phys. 135, 184111 (2011).

${ }^{14}$ P. Koskinen and V. Mäkinen, Comput. Mater. Sci. 47, 237 (2009).

${ }^{15}$ A. F. Oliveira, G. Seifert, T. Heine, and H. A. Duarte, J. Braz. Chem. Soc. 20, 1193 (2009).

${ }^{16}$ G. Seifert and J.-O. Joswig, WIREs: Comput. Mol. Sci. 2, 456 (2014).

${ }^{17}$ M. Gaus, Q. Cui, and M. Elstner, WIREs: Comput. Mol. Sci. 4, 49 (2014).

${ }^{18}$ D. Porezag, T. Frauenheim, T. Köhler, G. Seifert, and R. Kaschner, Phys. Rev. B 51, 12947 (1995)
${ }^{19}$ M. Elstner, D. Porezag, G. Jungnickel, J. Elsner, M. Haugk, T. Frauenheim, S. Suhai, and G. Seifert, Phys. Rev. B 58, 7260 (1998).

${ }^{20}$ T. A. Niehaus, S. Suhai, F. Della Sala, P. Lugli, M. Elstner, G. Seifert, and T. Frauenheim, Phys. Rev. B 63, 085108 (2001).

${ }^{21}$ D. Heringer, T. A. Niehaus, M. Wanko, and T. Frauenheim, J. Comput. Chem. 28, 2589 (2007).

${ }^{22}$ N. C. Handy and H. F. Schaefer III, J. Chem. Phys. 81, 5031 (1984).

${ }^{23}$ X. Wang, R. Q. Zhang, S. T. Lee, T. A. Niehaus, and T. Frauenheim, Appl. Phys. Lett. 90, 123116 (2007).

${ }^{24}$ Q. Wu, X.-H. Wang, T. Niehaus, and R.-Q. Zhang, J. Phys. Chem. C 118, 20070 (2014).

${ }^{25}$ R. Mitrić, U. Werner, M. Wohlgemuth, G. Seifert, and V. Bonačić-Koutecký, J. Phys. Chem. A 113, 12700 (2009).

${ }^{26}$ G. Fan, J. Liu, and G. He, Comput. Theor. Chem. 1023, 10 (2013).

${ }^{27}$ C. Camacho, T. A. Niehaus, K. Itami, and S. Irle, Chem. Sci. 4, 187 (2013).

${ }^{28}$ M. Elstner, Theor. Chem. Acc. 116, 316 (2006).

${ }^{29}$ Y. Yang, H. Yu, D. York, Q. Cui, and M. Elstner, J. Phys. Chem. A 111, 10861 (2007).

${ }^{30}$ M. Gaus, Q. Cui, and M. Elstner, J. Chem. Theory Comput. 7, 931 (2011).

${ }^{31}$ M. Elstner, J. Phys. Chem. A 111, 5614 (2007).

${ }^{32}$ M. Gaus, A. Goez, and M. Elstner, J. Chem. Theory Comput. 9, 338 (2013).

${ }^{33}$ P. Goyal, M. Elstner, and Q. Cui, J. Phys. Chem. B 115, 6790 (2011).

${ }^{34}$ P. Goyal, H.-J. Qian, S. Irle, X. Lu, D. Roston, T. Mori, M. Elstner, and Q. Cui, J. Phys. Chem. B 118, 11007 (2014).

${ }^{35}$ M. Gaus, X. Lu, M. Elstner, and Q. Cui, J. Chem. Theory Comput. 10, 1518 (2014).

${ }^{36}$ X. Lu, M. Gaus, M. Elstner, and Q. Cui, J. Phys. Chem. B 119, 1062 (2015).

${ }^{37}$ M. Kubillus, T. Kubař, M. Gaus, J. Řezáč, and M. Elstner, J. Chem. Theory Comput. 11, 332 (2015).

${ }^{38}$ C. Köhler, G. Seifert, U. Gerstmann, M. Elstner, H. Overhof, and T. Frauenheim, Phys. Chem. Chem. Phys. 3, 5109 (2001).

${ }^{39}$ G. Zheng, M. Lundberg, J. Jakowski, T. Vreven, M. J. Frisch, and K. Morokuma, Int. J. Quantum Chem. 109, 1841 (2009).

${ }^{40}$ F. Trani, G. Scalmani, G. Zheng, I. Carnimeo, M. J. Frisch, and V. Barone, J. Chem. Theory Comput. 7, 3304 (2011).

${ }^{41}$ J. F. Janak, Phys. Rev. B 18, 7165 (1978).

${ }^{42}$ E. R. Davidson, J. Comput. Phys. 17, 87 (1975).

${ }^{43}$ S. Hirata and M. Head-Gordon, Chem. Phys. Lett. 314, 291 (1999).

${ }^{44}$ D. W. Silverstein, N. Govind, H. J. J. van Dam, and L. Jensen, J. Chem. Theory Comput. 9, 5490 (2013).

${ }^{45}$ H. A. Witek, S. Irle, and K. Morokuma, J. Chem. Phys. 121, 5163 (2004).

${ }^{46}$ M. W. Schmidt, K. K. Baldridge, J. A. Boatz, S. T. Elbert, M. S. Gordon, J. J. Jensen, S. Koseki, N. Matsunaga, K. A. Nguyen, S. Su, T. L. Windus, M. Dupuis, and J. A. Montgomery, J. Comput. Chem. 14, 1347 (1993).

${ }^{47}$ T. Niehaus, M. Elstner, T. Frauenheim, and S. Suhai, J. Mol. Struct.: THEOCHEM 541, 185 (2001).

${ }^{48}$ See http://www.dftb.org for the dftb web site.

${ }^{49}$ L. Goerigk, J. Moellmann, and S. Grimme, Phys. Chem. Chem. Phys. 11, 4611 (2009).

${ }^{50}$ L. Goerigk and S. Grimme, J. Chem. Phys. 132, 184103 (2010).

${ }^{51}$ W. Humphrey, A. Dalke, and K. Schulten, J. Mol. Graphics 14, 33 (1996).

${ }^{52}$ A. Domínguez, B. Aradi, T. Frauenheim, V. Lutsker, and T. A. Niehaus, J. Chem. Theory Comput. 9, 4901 (2013).

${ }^{53}$ T. Kubar̆, Z. Bodrog, M. Gaus, C. Köhler, B. Aradi, T. Frauenheim, and M. Elstner, J. Chem. Theory Comput. 9, 2939 (2013).

${ }^{54}$ D. I. Kreller and P. V. Kamat, J. Phys. Chem. 95, 4406 (1991).

${ }^{55} \mathrm{~J}$. W. Ponder, TINKER: Software Tools for Molecular Design, 6.0 (Washington University School of Medicine, Saint Louis, MO, 2009).

${ }^{56}$ J. Wang, P. Cieplak, and P. A. Kollman, J. Comput. Chem. 21, 1049 (2000).

${ }^{57}$ A. K. Rappé, C. J. Casewit, K. S. Colwell, W. A. Goddard III, and W. M. Skiff, J. Am. Chem. Soc. 114, 10024 (1992).

${ }^{58}$ L. Zhechkov, T. Heine, S. Patchkovskii, G. Seifert, and H. A. Duarte, J. Chem. Theory Comput. 1, 841 (2005).

${ }^{59}$ L. Bernasconi, M. Sprik, and J. Hutter, J. Chem. Phys. 119, 12417 (2003).

${ }^{60}$ T. A. Niehaus and F. Della Sala, Phys. Status Solidi B 249, 237 (2012).

${ }^{61}$ V. Barone, I. Carnimeo, and G. Scalmani, J. Chem. Theory Comput. 9, 2052 (2013).

${ }^{62}$ K. Kitaura, E. Ikeo, T. Asada, T. Nakano, and M. Uebayasi, Chem. Phys. Lett. 313, 701 (1999).

${ }^{63}$ Y. Nishimoto, D. G. Fedorov, and S. Irle, J. Chem. Theory Comput. 10, 4801 (2014). 